\title{
ALTERAÇÕES CLÍNICAS E LABORATORIAIS ANTERIORES AO DESENVOLVIMENTO DO DELIRIUM TREMENS
}

\author{
PAULO BORIN"*, CLEUZA OLIVATTO DA SILVA **
}

\begin{abstract}
RESUMO - Duzentos e trinta pacientes alcoolistas crônicos do sexo masculino internados em hospital psiquiátrico para tratamento do alcoolismo foram examinados clínica e laboratorialmente dentro das primeiras 24 horas da internação. Treze pacientes que desenvolveram delirium tremens (DT) após a internação (grupo l) constituem a casuística deste estudo que visa avaliar a ocorrência de alteraçōes clínicas, hematológicas, bioquímicas hepáticas e eletrolíticas que antecedem ao aparecimento das manifestações do DT. Para análises comparativas foram estudados dois grupos de 26 pacientes cada um, constituídos de pacientes que não entraram em DT na internação atual mas com história pregressa de DT (grupo II) e pacientes aleatoriamente arrolados - amostra aleatória simples obtida empregando tabela de números aleatórios fornecida por computador - que nunca entraram em DT (grupo III). Os pacientes do grupo I apresentavam média de idade significativamente mais baixa e fisicamente encontravam-se em pior estado geral que os do grupo III. As frequências de taxas elevadas de aminotransferases e de hipomagnesemia foram significativamente maiores nos grupos I e II que no grupo III. Os valores plasmáticos médios das aminotransferases, especialmente da aspartato-aminotransferase, estavam significativamente mais elevados nos grupos I e II que no grupo III.
\end{abstract}

PALAVRAS-CHAVE: síndrome de abstinência do álcool, delirium tremens, clínica, bioquímica, alteraçōes pré-delirium tremens.

\section{Clinical and laboratorial alterations previous at the delirium tremens}

ABSTRACT - Thirteen alcoholic male patients that developed delirium tremens (DT) after admission in a psychiatric hospital for treatment of alcoholism (group I) had their clinical and laboratorial records examined. The laboratory samples were taken during the phase previous at the DT. Data on this group were compared to those of two other groups of alcoholics - 26 patients each - that did not develop DT in the present admission, with (group II) or without (group III) previous history of DT. The patients of group I had significantly lower average age and worse general conditions than the patients of group III. The frequency of elevated aminotransferases and hypomagnesemia was significantly higher in the group I and II than in the group III. The aminotransferases, especially the aspartate-aminotransferase, were significantly more elevated in the groups I and II.

KEY WORDS: alcohol withdrawal, delirium tremens, clinics, biochemistry, alterations previous at the delirium tremens.

A síndrome de abstinência do álcool (SAA) pode manifestar-se por quadros clínicos com diferentes graus de severidade. As características das manifestações e a faculdade de drogas simpaticolíticas de atenuar os sintomas sugerem que a hiperatividade adrenérgica é a geradora da SAA ${ }^{19,22,24}$. O delírio tremens (DT), caracterizado por tremor generalizado, acompanhado por distúrbios da percep̧̧ão com alucinações visuais ou auditivas, insônia, taquicardia, sudorese profusa, náuseas e vômitos, hipertermia, eventualmente convulsões tônico-clônicas e um estado confusional com agitação, constitui a consequência mais grave do declínio da concentração cerebral de etanol em

Hospital Espírita de Marilia, SP: * Professor Assistente de Clínica Médica da Faculdade de Medicina de Marilia, Médico Clínico do Hospital Espírita de Marília; ** Enfermeira do Hospital Espirita de Marlia. Aceite: 11-setembro-1996.

Dr. Paulo Borini - Rua Gabriel Monteiro da Silva 40 - 17501-150 Marilia SP - Brasil. 
alcoolistas crônicos. Os sintomas neurológicos da retirada do álcool mostram semelhança com aqueles vistos na hiponatremia ou intoxicação aquosa. Isto sugere que os alcoolistas mostram sinais de super-hidratação durante a retirada e que tais manifestações estariam relacionadas com edema cerebral $^{20}$. Dados obtidos através da ressonância magnética nuclear sugerem que no DT tanto a substância branca quanto a cinzenta do cérebro retêm água'

A incidência de DT tem variado, de acordo com diversos estudos, entre $4 \%$ e $13 \%$ dos alcoolistas crônicos ${ }^{7}$. Entretanto, a incidência e a prevalência deste distúrbio bem como suas relações com fatores de ordem demográfica, epidemiológica, clínica, bioquímica e eletrolítica não têm sido estudadas com frequência entre nós.

Este estudo tem dupla finalidade: (1) avaliar as alterações clínicas, hematológicas, bioquímicas hepáticas e eletroliticas presentes em pacientes na fase pré-delirio e (2) comparar as alteraçōes observadas nestes pacientes com as encontradas em alcoolistas que não desenvolveram DT na internação atual mas com e sem história de DT no passado.

\section{CASUISTICA E MÉtodos}

Dentre 230 pacientes alcoolistas, do sexo masculino, internados em hospital psiquiátrico durante um período de três meses, 18 (7,8\%) desenvolveram DT $^{7}$. Treze destes pacientes constituem parte da casuistica deste estudo (Grupo I). Cinco pacientes foram excluídos por já apresentarem quadro clínico de DT por ocasião da internação. A instalação do DT ocorreu dentro das primeiras 96 horas da internação. Alucinaçōes visuais e ou auditivas foram observadas em todos os pacientes. Agitação, sudorese profusa e tremores constituíram as outras manifestaçōes mais frequentes.

$\mathrm{Na}$ fase pré-delirio, todos pacientes foram submetidos a exames médico e laboratorial.

A anamnese foi realizada na forma de entrevista, empregando-se modelo estruturado, elaborado com a finalidade de obter-se, entre outras informaçōes, aquelas referentes a aspectos demográficos, epidemiológicos, manifestaçōes clínicas e psiquiátricas, internaçōes anteriores e padrões de alcoolismo ${ }^{3}$.

No exame físico considerou-se haver hepatomegalia quando o fígado estava palpável abaixo da reborda costal, quer na linha hemiclavicular direita quer na linha mediana, desde que o nível superior da macicez hepática não estivesse abaixo do quinto espaço intercostal direito. Considerou-se haver esplenomegalia não somente quando o baço estivesse palpável abaixo da reborda costal, mas também quando o nível superior da macicez detectada no espaço de Traube deslocava-se para baixo quando o paciente fazia apnéia inspiratória.

Os exames laboratoriais consistiram de análises hematimétricas e dosagens bioquímicas plasmáticas de proteínas (métodos do biureto e verde de bromocresol), bilirrubinas (método de Sims-Hom), aminotransferases (método Reitman e Frankel), fosfatase alcalina (método de Roy modificado, timolftaleína), glicemia de jejum (método enzima-oxidase) e eletrólitos - sódio e potássio (fotometria de chama), cálcio (método colorimétrico, cresolftaleína), fósforo (método de Basques-Lustosa) e magnésio (método de Basques-Lustosa, azul de molibdênio).

Para análise comparativa foram estudados, empregando-se a mesma metodologia, dois grupos de 26 alcoolistas cada. Um deles (Grupo II), formado por todos os pacientes que não apresentaram DT na internação atual mas que relatavam ter apresentado DT no passado e outro (Grupo III) formado por pacientes aleatoriamente arrolados - amostra aleatória simples obtida empregando-se tabela de números aleatórios fornecida por computador - que nunca apresentaram DT.

Nenhum paciente havia feito uso de medicação psicotrópica nos três meses anteriores à internação . A maioria tinha nivel baixo de escolaridade e baixa renda. ${ }^{3}$

Os dados sāo apresentados como média \pm desvio padrāo no texto e nas tabelas. Comparaçð̌es estatísticas foram feitas pelos testes qui-quadrado e t de Student para as variáveis qualitativas e quantitativas, respectivamente ${ }^{30}$. Adotou-se, para análise estatística, o nível de significância de $5 \%$.

\section{RESULTADOS}

\section{Aspectos demográficos e epidemiológicos}

A média de idade dos pacientes que desenvolveram DT na atual internaçăo foi mais baixa que as dos outros dois grupos, porém foi significativamente menor apenas em relação ao grupo de pacientes que nunca desenvolveram DT. A ocorrência de DT não guardou relaçāo com cor, estado civil e 
religiāo (Tabela 1). Cerca de $84,6 \%$ dos pacientes do grupo I tinham idades situadas abaixo de 40 anos, $69,2 \%$ com idades situadas entre 30 e 40 anos e igual número de pacientes $(15,4 \%)$ tinha idade abaixo de 30 e acima de 40 anos. No grupo III, apenas $50 \%$ dos pacientes tinham idades inferiores a 40 anos e 38,5\% tinham idades entre 30 e 40 anos (Tabela 2).

Não houve diferenças significativas entre os três grupos quanto ao número médio de internações - que pode ser considerado como indicativo do número de períodos de abstinência - nem quanto aos padrōes de alcoolismo ou uso concomitante de outras substâncias (Tabela 3).

A ocorrência de DT no Grupo I foi maior que no Grupo II quanto aos pacientes que estavam se internando pela primeira vez mas a diferença não foi significativa. Considerando o número total de pacientes com quadro de DT, independentemente de ter ele ocorrido no presente ou no passado, não houve diferenças significativas entre os Grupos I e II. Também as frequências médias de episódios de DT foram semelhantes para ambos os grupos (Tabela 3).

\section{Aspectos emocionais e comportamentais}

Os estados emocionais dos pacientes dos três grupos, tanto quando alcoolizados como quando sóbrios, não pareceram ter influenciado na ocorrência de DT (Tabela 4).

\section{Aspectos clínicos}

Excetuando a condição subjetiva de melhor "estado geral"dos pacientes do Grupo III, nenhum outro sintoma ou sinal clínico, em especial os indicativos de comprometimento do fígado, foi significativamente diferente entre os três grupos (Tabela 5).

Tabela 1. Características demográficas e epidemiológicas.

\begin{tabular}{lccc}
\hline & Grupo I & Grupo II & Grupo III \\
\hline Número de pacientes & 13 & 26 & 26 \\
Média de idade(anos) & $34,4 \pm 6,2 *$ & $39,1 \pm 8,5$ & $42,8 \pm 13,3^{*}$ \\
Limites de idades & $22 \cdot 48$ & $23-52$ & $24-73$ \\
Cor & & & \\
Branca & $10(76,9)$ & $19(73,1)$ & $24(92,4)$ \\
Preta & $2(15,4)$ & $5(19,2)$ & $1(3,8)$ \\
Parda & $1(7,7)$ & $2(7,7)$ & $1(3,8)$ \\
Estado civil & & & \\
Casado & $6(46,2)$ & $7(26,9)$ & $12(46,2)$ \\
Desquitado & $2(15,3)$ & $6(23,0)$ & $4(15,4)$ \\
Solteiro & $5(38,5)$ & $12(46,3)$ & $10(38,4)$ \\
Viúvo & 0 & $1(3,8)$ & 0 \\
Religiāo & & & $21(80,8)$ \\
Católica & $12(46,3)$ & $23(88,5)$ & $4(15,4)$ \\
Espirita & 0 & $3(11,5)$ & 0 \\
Protestante & $1(7,7)$ & 0 & $1(3,8)$ \\
Outra & 0 & 0 & $9(34,6)$ \\
Procedência & & $9(34,6)$ & $17(65,4)$ \\
Marília & $4(30,8)$ & $17(65,4)$ & \\
Outras cidades & $9(69,2)$ & 0 & \\
\hline
\end{tabular}

Grupo I, delirium tremens na internação atual; Grupo II, delirium tremens no passado; Grupo III, nunca apresentou delirium tremens; os números entre parênteses indicam percentuais; teste t de Student * $p<0,05$. 
Tabela 2. Faixas etárias dos pacientes dos três grupos estudados.

\begin{tabular}{cccccccccc}
\hline & \multicolumn{3}{c}{ Grupo I } & \multicolumn{3}{c}{ Grupo II } & \multicolumn{3}{c}{ Grupo III } \\
\hline Faixas de idades & n. & $\%$ & $\% \mathrm{c}$ & n. & $\%$ & $\% \mathrm{c}$ & n. & $\%$ & $\% \mathrm{c}$ \\
\hline $20-24$ anos & 1 & 7,7 & 7,7 & 1 & 3,8 & 3,8 & 1 & 3,8 & 3,8 \\
$25-29$ anos & 1 & 7,7 & 15,4 & 3 & 11,5 & 15,3 & 2 & 7,7 & 11,5 \\
$30-34$ anos & 4 & 30,8 & 46,2 & 3 & 11,5 & 26,8 & 7 & 27,0 & 38,5 \\
$35-40$ anos & 5 & 38,4 & 84,6 & 8 & 30,8 & 57,6 & 3 & 11,5 & 50,0 \\
$41-44$ anos & 1 & 7,7 & 92,3 & 4 & 15,4 & 73,0 & 4 & 15,4 & 65,4 \\
$>45-<50$ anos & 1 & 7,7 & 100 & 7 & 27,0 & 100 & 9 & 34,6 & 100 \\
\hline
\end{tabular}

Legenda: ver Tabela $1 . \% \mathrm{c}$, percentual cumulativo.

Tabela 3. Padröes de alcoolismo. Internações e ocorrências de delirium tremens.

\begin{tabular}{lccc}
\hline & Grupo I & Grupo II & Grupo III \\
\hline $\begin{array}{l}\text { Padrões de alcoolismo } \\
\text { tempo de alcoolismo (anos) }\end{array}$ & $18,6 \pm 7,5$ & & \\
consurno (gramas/dia) & $342 \pm 154$ & $22,0 \pm 7,8$ & $25,8 \pm 13,3$ \\
Internaçð̃es psiquiátricas & & $459 \pm 224$ & $366 \pm 257$ \\
média & $4,8 \pm 5,0$ & & \\
primeira internação & $4(30,8)$ & $9,0 \pm 12,8$ & $3,4 \pm 2,7$ \\
Delirium tremens & 13 & $3(11,5)$ & $8(30,8)$ \\
atual & 11 & & 0 \\
no passado & 24 & 42 & 0 \\
total & $1,8 \pm 1,2$ & 42 & 0 \\
média de DT & $13(100)$ & $1,6 \pm 1,2$ & 0 \\
Tabagismo & $1(7,7)$ & $24(92,3)$ & $21(80,8)$ \\
Outras drogas & $3(11,5)$ & $3(11,5)$ \\
\hline
\end{tabular}

Legenda: ver Tabela 1 .

\section{Aspectos laboratoriais}

O número de pacientes do Grupo I com aminotransferases aumentadas e com hipomagnesemia foi significativamente maior que o do Grupo III (Tabelas 6 e 7 ).

Os valores médios das aminotransferases, especialmente da aspartato-aminotransferase, estavam significativamente mais elevados nos Grupos I e II que no Grupo III. A taxa média de magnésio estava mais baixa no Grupo I que no Grupo III mas sem significância estatística. Diferença significativa na taxa média de magnésio ocorreu entre o Grupo II e os outros dois grupos (Tabela 6).

Os grupos mostraram-se homogêneos com relação às frequências das alterações e valores médios das demais provas laboratoriais, tanto bioquímicas como hematimétricas (Tabelas 6, 7 e 8).

\section{DISCUSSĀO}

Em nosso estudo, delirium tremens ocorreu em $7,8 \%$ dos alcoolistas crônicos internados. Estes índices estão situados dentro dos limites das taxas de incidência descritas em estudos prévios que têm examinado populações semelhantes ${ }^{9,13,23,26}$. 
Tabela 4. Características emocionais.

\begin{tabular}{|c|c|c|c|c|c|c|}
\hline & \multicolumn{3}{|c|}{ Acoolizado } & \multicolumn{3}{|c|}{ Sóbrio } \\
\hline & \multicolumn{3}{|c|}{ Grupos } & \multicolumn{3}{|c|}{ Grupos } \\
\hline & I & II & III & I & I & III \\
\hline Agressivo & $4(30,8)$ & $12(46,2)$ & $9(34,6)$ & $1(7,7)$ & $2(7,7)$ & 0 \\
\hline Deprimido & $2(15,4)$ & $2(7,7)$ & $3(11,5)$ & $3(23,1)$ & $3(11,5)$ & $3(11,5)$ \\
\hline Agitado & $1(7,7)$ & $2(7,7)$ & $5(19,2)$ & $1(7,7)$ & 0 & 0 \\
\hline Inalterado & $6(46,2)$ & $10(38,5)$ & $11(42,3)$ & $8(61,5)$ & $21(80,7)$ & $19(73,1)$ \\
\hline
\end{tabular}

Legenda: ver Tabela 1.

Tabela S. Aspectos clínicos.

\begin{tabular}{lccc}
\hline & Grupo I & Grupo II & Grupo III \\
\hline $\begin{array}{l}\text { Estado geral } \\
\text { bom }\end{array}$ & $3(23,1)^{*}$ & $10(38,5)$ & $14(53,8)^{*}$ \\
regular & $9(69,2)$ & $13(50,0)$ & $11(42,3)$ \\
mau & $1(7,7)$ & $3(11,5)$ & $1(3,8)$ \\
Alimentação & & & \\
regular & $4(30,8)$ & $6(46,2)$ & $9(34,6)$ \\
irregular & $9(69,2)$ & $20(76,9)$ & $17(65,4)$ \\
Manifestaçס̄es clínicas & & & \\
caimbras & $7(53,8)$ & $14(53,8)$ & $9(34,6)$ \\
parestesias & $5(38,5)$ & $8(30,8)$ & $9(34,6)$ \\
convulsões & $4(30,8)$ & $8(30,8)$ & $5(19,2)$ \\
icterícia & $1(7,7)$ & $2(7,7)$ & 0 \\
pseudo-ictericia & $5(38,5)$ & $11(42,3)$ & $15(57,7)$ \\
aranhas vasculares & $3(23,1)$ & $9(34,6)$ & $3(11,5)$ \\
edema & $1(7,7)$ & $1(3,8)$ & $1(3,8)$ \\
circulação colateral & 0 & $1(3,8)$ & $1(3,8)$ \\
ascite & 0 & $1(3,8)$ & 0 \\
ginecomastia & 0 & $1(3,8)$ & 0 \\
hepatomegalia & $8(61,5)$ & $22(84,6)$ & $15(57,7)$ \\
esplenomegalia & $12(92,3)$ & $20(76,9)$ & $24(92,3)$ \\
\hline
\end{tabular}

Legenda: ver Tabela 1. Teste do qui-quadrado: ${ }^{*} p<0,05$

Os eletrencefalogramas dos pacientes dos três grupos que apresentaram crise convulsiva foram normais.

A existência de fator biológico constitucional predisponente para o DT tem causado controvérsias. Os que não o admitem se apoiam na observação de que os pacientes que desenvolveram DT em um dos períodos de abstinência não necessariamente irão apresentá-lo em outros períodos. Por outro lado, os que admitem existir determinadas condiçōes constitucionais para a ocorrência de DT argumentam que este se desenvolve quando a elas se associam fator ou fatores circunstanciais desencadeantes. Distúrbios hormonais, metabólicos e hidro-eletrolíticos que ocorrem na SAA, associados a outras condiçōes - idade, intensidade e tempo de consumo de bebida alcóolica, ocorrência de convulsões e infecções concomitantes - seriam elementos precipitantes do $\mathrm{DT}^{10.14 .18 .24 .31}$. 
Tabela 6. Características bioquimicas.

\begin{tabular}{|c|c|c|c|c|}
\hline & Grupo I & Grupo II & Grupo III & Referência \\
\hline \multicolumn{5}{|l|}{ Proteínas } \\
\hline \multicolumn{5}{|l|}{ Albumina } \\
\hline média (g\%) & $3,5 \pm 0,7$ & $3,3 \pm 0,6$ & $3,4 \pm 0,6$ & $3,5-4,8$ \\
\hline hipoalbuminemia & $5(38,5)$ & $18(69,2)$ & $15(57,7)$ & \\
\hline \multicolumn{5}{|l|}{ Globulinas } \\
\hline média ( $\mathrm{g} \%)$ & $4,1 \pm 1,6$ & $4,0 \pm 0,8$ & $3,5 \pm 1,0$ & $2,6-3,1$ \\
\hline hiperglobulinemia & $10(76,9)$ & $21(80,8)$ & $18(69,2)$ & \\
\hline \multicolumn{5}{|l|}{ Bilimubinas } \\
\hline total (mg\%) & $1,0 \pm 0,4$ & $1,0 \pm 0,4$ & $0,9 \pm 0,2$ & $0,3-1,2$ \\
\hline direta $(\mathrm{mg} \%)$ & $0,6 \pm 0,3$ & $0,6 \pm 0,2$ & $0,5 \pm 0,1$ & $0,2-0,4$ \\
\hline \multicolumn{5}{|l|}{ Aminotransferases } \\
\hline \multicolumn{5}{|l|}{ AST } \\
\hline média (UI) & $15,2 \pm 5,2 *$ & $17,2 \pm 8,7$ & $12,3 \pm 3,4^{*}$ & até 12 \\
\hline elevada & $9(69,2) *$ & $16(61,5)$ & $8(30,7) *$ & \\
\hline \multicolumn{5}{|l|}{ ALT } \\
\hline média (UI) & $9,1 \pm 2,7^{*}$ & $9,9 \pm 5,0$ & $7,1 \pm 1,2 *$ & até 12 \\
\hline elevada & $2(15,4) *$ & $5(19,2)$ & $0^{*}$ & \\
\hline \multicolumn{5}{|l|}{ Fosfatase alcalina } \\
\hline média (U) & $50,9 \pm 16,8$ & $47,2 \pm 22,4$ & $45,4 \pm 21,2$ & $13-43$ \\
\hline elevada & $9(69,2)$ & $12(46,2)$ & $12(46,2)$ & \\
\hline Glicemia (mg\%) & $83,1 \pm 22,2$ & $76,1 \pm 20,3$ & $79,0 \pm 27,3$ & $60-110$ \\
\hline hipoglicemia & $1(7,7)$ & $3(11,5)$ & $4(15,4)$ & \\
\hline hiperglicemia & $1(7,7)$ & $2(7,7)$ & $2(7,7)$ & \\
\hline
\end{tabular}

Legenda: ver Tabela 1. Testes do qui-quadrado e t de Student: $* p<0,05$.

AST, aspartato-aminotransferase; ALT, alanino-aminotransferase.

Tabela 7. Alterações eletroliticas.

\begin{tabular}{lcccc}
\hline & Grupo I & Grupo II & Grupo III & Referências \\
\hline $\begin{array}{l}\text { Sódio } \\
\text { média (mEq/1) }\end{array}$ & $138,6 \pm 5,7$ & $138,8 \pm 4,2$ & $139,5 \pm 5,6$ & $138-146$ \\
hiponatremia & $2(15,4)$ & $9(34,6)$ & $7(26,9)$ & \\
$\begin{array}{l}\text { Potássio } \\
\text { média (mEq/1) }\end{array}$ & & & \\
hipopotassemia & $4,3 \pm 0,8$ & $4,3 \pm 0,8$ & $4,1 \pm 0,6$ & $3,6-5,2$ \\
$\begin{array}{l}\text { Cálcio } \\
\text { média (mg\%) }\end{array}$ & $3(23,1)$ & $5(19,2)$ & $4(15,4)$ & \\
$\quad$ hipocalcemia & $9,4 \pm 1,0$ & $9,8 \pm 1,0$ & $9,5 \pm 0,9$ & $9-11$ \\
Fósforo & $4(30,8)$ & $5(19,2)$ & $9(34,6)$ & \\
média (mg\%) & & & & \\
hipofosfatemia & $3,8 \pm 1,0$ & $3,9 \pm 0,9$ & $3,7 \pm 0,9$ & $2,5-4,8$ \\
Magnésio & $1(7,7)$ & $2(7,7)$ & $3(11,5)$ & \\
média (mg\%) & & & & $1,9-2,5$ \\
hipomagnesemia & $1,5 \pm 0,3$ & $1,9 \pm 0,4$ & $1,7 \pm 0,4$ & \\
\hline
\end{tabular}

Legenda: ver Tabela 1. Teste do qui-quadrado: ${ }^{*} p<0,05$ 
Tabela 8. Aspectos hematimétricos.

\begin{tabular}{lrcc}
\hline & Grupo I & Grupo II & Grupo III \\
\hline Anemia & $3(23,1)$ & $8(30,8)$ & $5(19,2)$ \\
$\begin{array}{l}\text { Reticulócitos } \\
\text { normal }\end{array}$ & $2(15,4)$ & $8(30,7)$ & \\
reticulocitose & $2(15,4)$ & $6(23,1)$ & $12(46,1)$ \\
reticulopenia & $9(69,2)$ & $10(38,5)$ & $2(7,8)$ \\
Leucograma & & & $12(46,1)$ \\
normal & $9(69,2)$ & $20(76,9)$ & \\
leucocitose & $5(38,5)$ & $6(23,1)$ & $6(76,9)$ \\
contagem diferencial & & & $6(23,1)$ \\
$\quad$ neutrofilia & $5(38,5)$ & $8(30,8)$ & $6(23,1)$ \\
$\quad$ linfocitose & $4(30,8)$ & $14(53,8)$ & $12(46,2)$ \\
$\quad$ eosinofilia & $9(69,2)$ & $10(38,5)$ & $14(53,8)$ \\
Plaquetas & & & $25(96,2)$ \\
$\quad$ normal & $11(84,6)$ & $24(92,3)$ & $1(3,8)$ \\
plaquetocitose & $2(15,4)$ & $1(3,8)$ & \\
\hline
\end{tabular}

Legenda: ver Tabela 1.

Embora o DT possa ou não ocorrer em um mesmo indivíduo em diferentes períodos de abstinência, a maioria das observaçōes deste estudo torna plausível admitir a existência de fator constitucional predisponente: (1) A maioria dos pacientes nunca apresentara manifestaçōes desta complicaçăo da SAA embora as frequências de períodos de abstinência - representados pelo número de internaçōes - e os padrōes de alcoolismo deles tenham sido semelhantes aos dos que desenvolveram DT; (2) O número total de episódios de DT entre os dois grupos de pacientes que o apresentaram nas várias internações a que se submeteram foram muito semelhantes.

A idade dos indivíduos parece constituir fator influente na ocorrência do DT. A média de idade do grupo de pacientes que desenvolveram DT foi significativamente mais baixa que a dos demais grupos e cerca de $70 \%$ dos pacientes que entraram em DT na internação atual tinham idades situadas entre 30 e 40 anos. Já foi observado que o DT raramente ocorre em pessoas abaixo dos 30 anos de idade ${ }^{8}$, embora a taxa de ocorrência em $15 \%$ dos indivíduos com menos de 30 anos, encontrada neste estudo, não permite falar em raridade.

Ainda no tocante a idade, a verificação de que indivíduos com idades acima de 40 anos mais dificilmente entram em DT poderia estar relacionada com observações realizadas em dois outros estudos envolvendo os mesmos pacientes deste estudo.Em cerca de $80 \%$ deles o alcoolismo já estava definitivamente estabelecido antes de completarem 20 anos de idade - por volta dos 18 anos ${ }^{5}$ - e que depois de aproximadamente 30 anos de consumo ocorre uma sensível redução na quantidade de álcool ingerida diariamente ${ }^{6}$, admitida como sendo decorrente de uma queda na tolerância por mudanças no metabolismo do álcool de natureza não definida ${ }^{27}$. Cerca de $50 \%$ dos pacientes que nunca haviam desenvolvido DT tinham idades superiores a 40 anos e tempo médio de alcoolismo próximo de 30 anos.

Estudos retrospectivos de pacientes que desenvolveram DT apontam como possíveis prognosticadores o uso diário e intenso do álcool, a história pregressa de DT e a ocorrência de convulsōes quando da retirada do álcool ${ }^{10}$. Nossas observações são concordantes apenas quanto à história pregressa de DT, pois $84,6 \%$ dos indivíduos que entraram em DT na internação atual já o haviam apresentado no passado. 
O uso intenso e constante de álcool, bem como a ocorrência de crises convulsivas, não parecem ser fatores de importância quer prognóstica quer desencadeadora de DT. $O$ padrão de alcoolismo dos pacientes que nunca apresentaram DT não diferiu significativamente daquele dos pacientes que apresentaram DT no presente e no passado e crises convulsivas ocorreram com igual frequência nos três gnupos estudados. Focos epileptógenos não foram observados nos eletrencefalogramas dos pacientes que apresentaram crises convulsivas, o que está em concordância com as observações de outros autores $^{12,32}$.

Executando o fato de que os indivíduos que entraram em DT se apresentavam em piores condições gerais, as alteraçōes clínicas e os estados emocionais dos pacientes, quer quando alcoolizados quer quando sóbrios, ocorreram com igual frequência nos três grupos, o que sugere não serem eles fatores relevantes propiciadores de DT.

A ausência de diferenças significativas entre os três grupos quanto a regularidade alimentar e quanto a presença de anemia, bem como a não existência de diferenças na ocorrência de leucocitose e na velocidade de hemossedimentação, sugerem que deficiências vitamínicas e infecções clinicamente inaparentes não constituíram fatores desencadeadores de DT.

Têm-se investigado interrelações entre diferentes sintomas de disfunção cerebral e hepática na SAA. Acredita-se que a natureza dos fatores que produzem DT é de índole metabólica. Mesmo existindo controvérsias quanto ao significado, alteraçōes patológicas, tanto de natureza degenerativa como inflamatória, podem ser vistas no cérebro e no fígado. Alguns estudos apontam para uma ausência de correlação significativa, conduzindo à hipótese de que o cérebro e o fígado, no mesmo indivíduo, reagem diferentemente ao álcool" ${ }^{11}$. Outros indicam uma associação entre as reações da retirada do álcool e anormalidades da função hepática ${ }^{33}$.

Foi demonstrado que as catecolaminas, elevadas durante a SAA, podem alterar o fluxo sanguíneo hepático e aumentar o consumo de oxigênio pelo figado. A ampla gama da resposta nervosa simpática na síndrome poderia explicar algumas diferenças na susceptibilidade individual à lesão hepática ${ }^{15}$. Neste estudo, as diferenças observadas no grau e na frequência das alteraçōes das aminotransferases dos pacientes que desenvolveram DT na intemação atual ou que relatavam já ter apresentado DT no passado relativamente àqueles que nunca apresentaram DT parecem indicar que a agressão hepática não seria um componente subjacente no desenvolvimento patogenético do DT. No máximo, poder-se-ia admitir, nestes indivíduos, haver maior susceptibilidade hepática e cerebral à agressão promovida pelo álcool.

Em estudo sobre as ações interativas do álcool e das substâncias presentes na fumaça do cigarro observamos que os alcoolistas tabagistas apresentavam maior facilidade para intoxicarem-se e maior suscetibilidade para desenvolverem DT que os alcoolistas não tabagistas e que as substâncias contidas na fumaça do tabaco potencializam a ação tóxica do álcool não só sobre o fígado como também sobre outras estruturas ${ }^{4}$.

As alteraçōes eletroliticas observadas nos vários estudos de pacientes com DT têm sido diferentes não só quanto ao eletrólito alterado como também quanto ao significado da alteração. Hipopotassemia e hipomagnesemia têm sido as alterações mais frequentemente encontradas em alcoolistas crônicos que desenvolveram $\mathrm{DT}^{2,14,18,29}$. Alteração significativa no nível de cálcio foi observada em um dos estudos ${ }^{17}$.

Alguns autores acham improvável a existência de uma relação causal específica entre hipomagnesemia e DT ${ }^{16}$, bem como não conseguem encontrar uma clara associação de hipomagnesemia e hipopotassemia como geradoras de manifestações específicas da SAA ${ }^{28}$. Em nosso estudo, observamos que o número de pacientes com hipomagnesemia era significativamente maior no grupo que desenvolveu DT que nos outros dois grupos controles. Também, as taxas médias de magnésio plasmático eram maiores nos pacientes dos grupos controles, embora a diferença fosse 
significativa apenas entre o grupo com DT na internação atual e o grupo que não apresentou DT no presente mas tinha história de DT no passado. Poderia ocorrer com relaçāo ao magnésio o que tem sido admitido para o potássio. Alguns autores admitem haver uma relação entre a intensidade das manifestações da SAA e o grau da hipopotassemia ${ }^{21,33}$.

Entretanto, outros autores sugerem que a hipopotassemia é apenas uma manifestação secundária da hiperatividade adrenérgica que ocorre no $\mathrm{DT}^{25}$.

Nossos resultados não parecem indicar haver relação entre hipomagnesemia e hipopotassemia, pois as frequências de alterações e as médias plasmáticas do potássio, diferentemente das do magnésio, foram semelhantes nos três grupos.

Algumas de nossas observaçōes não são concordantes com aquelas feitas por outros autores. Porém, o pequeno número de pacientes que constitui a casuistica deste estudo faz com que nossos resultados sejam confirmados por outras observações.

Dos 18 pacientes que desenvolveram DT, durante o periodo de realização deste estudo, 3 $(16,7 \%)$ apresentaram grave deterioração de suas condições físicas e tiveram que ser transferidos para unidade de terapia intensiva de hospital clínico. A morbidade e a mortalidade ainda prevalente entre estes pacientes, bem como a identificação mais precisa dos mecanismos de desenvolvimento e dos fatores predisponentes ou precipitantes, estão a exigir maior número de estudos sobre esta manifestação da SAA.

Agradecimentos - Agradecemos o apoio recebido da Diretoria do Hospital Espirita de Marilia para a realização deste trabalho e ao Prof. Dr. Romeu Cardoso Guimarães, pela revisão do texto.

\section{REFEREENCIAS}

1. Besson JA, Glen AI, Foreman EI, MacDonald A, Smith FW, Hutchison JM, Mallard JR, Ashcroft GW. Nuclear magnetic resonance observations in alcoholic cerebral disorders and the role of vasopressin. Lancet 1981;2:923-924.

2. Blay SL, Ferraz MPT, Calil HM, Novo NF. Alteraçōes eletrolíticas plasmáticas em pacientes alcoólatras crônicos com e sem delirium tremens. Acta Psiquiatr Psicol Amer Lat 1981;27:311-314.

3. Borini P. Anamnese psiquiátrica e clínica, exame físico e exames complementares estruturados e informatizados para aplicaçāo em alcoolistas. J Bras Psiq 1990;39:250-265.

4. Borini P. Interaçăo álcool-fumo na agressāo hepática. Rev Hosp Clin Fac Med Sāo Paulo 1995; 50:259-263.

5. Borini P, Silva CO. Aspectos demográficos, epidemiológicos e sociais do alcoolismo : uma análise de alcoolistas internados em hospital psiquiátrico. Rev ABP/APAL 1989;11:89-96.

6. Borini P, Silva CO. Análise de alguns aspectos do padrāo de alcoolismo e suas implicaçōes terapêuticas. Rev ABP-APAL 1991;13:105-111.

7. Borini P, Silva CO. Tratamento de alcoolistas em regime de internaçāo hospitalar: avaliação dos resultados. J Bras Psiq 1993; 42:149-155.

8. Chafetz ME. Addiction.III : Alcoholism. In Freedman AM, Kaplan HI (eds). Comprehensive textbook of psychiatry. Baltimore: Williams \& Wilkins, 1967:1011-1026.

9. Cordeiro MAP. Incidência do alcoolismo: estudos estatísticos. Considerações sociogenéticas. Rev Bras Saúde Mental 1969;13:77-97.

10. Cushman P Jr. Delirium tremens: update on an old disorder. Postgrad Med 1987; 82:117-122.

11. Elton M. Do brain damage and liver disease interrelate in advanced abuser of alcohol? Acta Psychiatr Scand 1983; 68:437-444.

12. Fernandes LNT. Complicaçōes neurológicas do alcoolismo. In Ramos SP et al. (eds). Alcoolismo hoje. Porto Alegre: Artes Medicas, 1987:84-96.

13. Finlayson RE, Hurt RD, Davis $\mathrm{L} J \mathrm{Jr}$, Morse RM. Alcoholism in elderly persons: a study of the psychiatric and psychosocial features of 216 inpatients. Mayo Clin Proc 1988;63:761-766.

14. Flink EB. Magnesium deficiency in alcoholism. Alcohol Clin Exp Res 1986;10:590-594.

15. Hadengue A, Moreau R, Lee SS, Gaudin C, Rueff B, Lebrec D. Liver hypermetabolism during alcohol withdrawal in humans: role of sympathetic overactivity. Gastroenterology 1988;94:1047-1052.

16. Jermain DM, Crismon ML, Nisbet RB. Controversies over the use of magnesium sulfate in delirium tremens. Ann Pharmacother $1992 ; 26: 650-652$.

17. Jost A, Hermle L, Spitzer M, Oepen G. Clinical and laboratory differentiation of alcohol withdrawal syndrome ("predelirium") and alcoholic delirium. Psychiatr Prax 1992;19:16-22.

18. Kaysen G, Noth RM. Efeitos do álcool sobre a pressāo arterial e os eletrólitos. In Geokas M (ed). Simpósio sobre álcool etílico e enfermidades associadas: Clin Med Am Norte 1984;1:235-262. 
19. Kemperman CJ, Kuilman M, Njio LK. A retrospective and explorative study of hypokalemia in psychiatric disorders : a beta 2-receptor related. Eur Arch Psychiatry Neurol Sci 1988;237:161-165.

20. Lambie DG. Alcoholic brain damage and neurological symptoms of alcohol withdrawal- manifestations of overhydration. Med Hypothesis 1985; 16:377-388.

21. Laso FJ, Gonzalez-Buitrago JM, Martin-Ruiz C, Vicens E, Moyano JC. Inter-relationship between serum potassium and plasma catecholamines and 3': 5 ' cyclic monophosphate in alcochol withdrawal. Drug Alcochol Depend 1990;26:183-188.

22. Linnoila A, Mefford J, Nutt D, Adinoff B. Alcochol withdrawal and nor-adrenergic function. Ann Intern Med 1987; 107:875-889.

23. Lundqvist G. A comparative study of pathogenesis, course and prognosis of delirium tremens. Acta Psychiatr Scand $1961 ; 36: 443$.

24. Manhem P, Nilss on LH, Moberg AL, Wadstein J. Hokfelt B. Alcohol withdrawal : effects of clonidine treatment on sympathetic activity, the renin-aldosterone system, and clinical symptoms. Alcohol Clin Exp Res 1985;9:238-243.

25. Nardoni A, Copetti R, Baldissera S, Busettini G, Cella R, Nador G. Hypopotassemia during delirium tremens: pathogenesis and clinical significance. Minerva Med 1982;73:3059-3062.

26. Nordstrom G, Berglund M. Delirium tremens : a prospective long-term follow-up study. J Stud Alcohol 1988;49:178-185.

27. Parry RA. Alcoholism and drug misuse. In Forrest $A$ (ed). Companion to psychiatric studies. Vol. 2. Edinburgh: Churchill Livingstone, 1973:116-137.

28. Ragland G. Electrolyte abnormalities in the alcoholic patient. Emerg Med Clin North Am 1990;8:761-773.

29. Schmickaly R, Nickel B, Jarisch M, Kursawe HK, Sachs E, Karson A. Electrolyte disorders, EEG changes and epileptic seizures in alcohol withdrawal delirium. Psychiatr Neurol Med Psychol Leipz 1989;41:722-729.

30. Sounis E. Bioestatistica.Ed. 3. Rio de Janeiro: Ateneu, 1985:206-234.

31. Trabert W, Caspari D, Bernhard P, Biro G. Inappropriate vasopressin secretion in severe alcohol withdrawal. Acta Psychiatr Scand 1992;85:376-379.

32. Victor $M$, Brausch $C$. The role of abstinence in the genesis of alcoholic epilepsy. Epilepsia 1967; 8:1-20.

33. Watson WS, Lawson PM, Beattie AD. The effect of acute alcohol withdrawal on the serum potassium and total body potassium in heavy drinkers. Scott Med J I984; 29:222-226. 